\title{
Nucleotide Analogue-Related Proximal Renal Tubular Dysfunction during Long-Term Treatment of Chronic Hepatitis B: A Cross-Sectional Study
}

\author{
Abhasnee Sobhonslidsuk, ${ }^{1}$ Jirachaya Wanichanuwat, ${ }^{1}$ Pawin Numthavaj, ${ }^{2}$ \\ Areepan Sophonsritsuk, ${ }^{3}$ Supanna Petraksa, ${ }^{1}$ Alongkorn Pugasub, ${ }^{4}$ Paisan Jittorntam, ${ }^{4}$ \\ Anucha Kongsomgan, ${ }^{5}$ Sittiruk Roytrakul, ${ }^{6}$ and Bunyong Phakdeekitcharoen ${ }^{7}$ \\ ${ }^{1}$ Division of Gastroenterology and Hepatology, Department of Medicine, Faculty of Medicine, Ramathibodi Hospital, \\ Mahidol University, Bangkok 10400, Thailand \\ ${ }^{2}$ Section for Clinical Epidemiology and Biostatistics, Faculty of Medicine, Ramathibodi Hospital, Mahidol University, \\ Bangkok 10400, Thailand \\ ${ }^{3}$ Department of Obstetrics and Gynecology, Faculty of Medicine, Ramathibodi Hospital, Mahidol University, Bangkok 10400, Thailand \\ ${ }^{4}$ Office of Research Academic and Innovation, Faculty of Medicine, Ramathibodi Hospital, Mahidol University, \\ Bangkok 10400, Thailand \\ ${ }^{5}$ Department of Pathology, Faculty of Medicine, Ramathibodi Hospital, Mahidol University, Bangkok 10400, Thailand \\ ${ }^{6}$ National Center for Genetic Engineering and Biotechnology, Pathumthani 12120, Thailand \\ ${ }^{7}$ Division of Nephrology, Department of Medicine, Faculty of Medicine, Ramathibodi Hospital, Mahidol University, \\ Bangkok 10400, Thailand
}

Correspondence should be addressed to Abhasnee Sobhonslidsuk; abhasnee.sob@mahidol.ac.th

Received 7 March 2016; Accepted 9 October 2016

Academic Editor: Fabio Marra

Copyright (C) 2016 Abhasnee Sobhonslidsuk et al. This is an open access article distributed under the Creative Commons Attribution License, which permits unrestricted use, distribution, and reproduction in any medium, provided the original work is properly cited.

\begin{abstract}
Background. There have been few reports of nucleotide analogue-related renal tubular dysfunction (RTD) in CHB patients. We assessed the prevalence and presentation of nucleotide analogue-related proximal RTD. Methods. A cross-sectional study was performed in CHB patients taking nucleotide analogues. Inclusion criteria were patients who were on adefovir or tenofovir as mono- or add-on therapy with lamivudine $(\mathrm{LAM})>1$ year. Serum and urine were collected. Fractional excretion of phosphate $\left(\mathrm{FEPO}_{4}\right)$, uric acid (FEUA), and potassium was calculated. Renal losses were defined based on the criteria: protein (24-hour urine protein $>150 \mathrm{mg}$ ), glucose (glycosuria with normoglycemia), phosphate $\left(\mathrm{FEPO}_{4}>18 \%\right)$, uric acid $(\mathrm{FEUA}>15 \%)$, potassium (renal potassium losses with hypokalemia), and bicarbonate (normal gap acidosis). Subclinical and overt proximal RTD were defined when 2 and $\geq 3$ criteria presented. Results. Ninety-two patients were enrolled. The mean duration of nucleotide analogue taking was $55.1 \pm 29.6$ months. Proximal RTD was found in $24(26.1 \%)$ patients (subclinical $15(16.3 \%)$ and overt $9(9.8 \%)$ ). The severity of RTD was associated with the duration of nucleotide analogue $(P=0.01)$. Conclusions. The prevalence of proximal RTD in CHB patients taking nucleotide analogues was $26 \%$. The severity of RTD was associated with the treatment duration. Comprehensive testing is necessary for early detecting nucleotide analogue-related nephrotoxicity.
\end{abstract}

\section{Introduction}

Over 350 million people around the world have been diagnosed with chronic hepatitis B (CHB) [1]. Progression of hepatitis $B$ virus (HBV) related chronic liver diseases depends on host and viral factors [1]. Risks of cirrhosis and hepatocellular carcinoma development increase significantly along with increase in baseline HBV viral load [2,3]. In patients infected with $\mathrm{HBV}$, long-term treatment with antiviral drugs such as lamivudine (LAM) slows disease progression by reducing the risk and incidence of hepatic decompensation and 
hepatocellular carcinoma until HBV resistant strains emerge [4]. Nucleoside and nucleotide analogues with a strong potency and high genetic barrier such as entecavir (ETV) and tenofovir disoproxil fumarate (TDF) are recommended as the first-line therapy of $\mathrm{CHB}$ [5-7]. When drug resistant $\mathrm{HBV}$ develops or incomplete virological response occurs, following the use of a low genetic barrier nucleoside analogue (e.g., LAM), a switch from LAM to TDF, or adding adefovir dipivoxil (ADV) or TDF to LAM is recommended [5, 8, 9]. ADV, a nucleotide analogue of adenosine monophosphate, was approved for CHB treatment in 2002 [8, 9]. It was followed by an approval of TDF in naïve and LAM resistant $\mathrm{CHB}$ in $2008[5,6,9]$. TDF is structurally similar to ADV but has a more potent antiviral effect on $\operatorname{HBV}[9,10]$.

A dose of $30 \mathrm{mg}$ daily of $\mathrm{ADV}$ has been shown in a randomized trial to be associated with mild renal dysfunction and hypophosphatemia, whereas a $10 \mathrm{mg}$ daily dose has been shown to carry no such risk [11]; therefore, the $10 \mathrm{mg}$ dose has been chosen to be the recommended daily dose of ADV in CHB treatment. In clinical practice, TDF has been believed to be safer in terms of nephrotoxicity than ADV [12], and this has been supported by previous studies which showed that TDF was associated with a low incidence of renal dysfunction [13-15]. On the other hand, subsequent small studies on the safety of ADV and TDF have shown that longterm treatment with ADV or TDF could potentially cause renal dysfunction, hypophosphatemia, and impaired renal tubular phosphate reabsorption [16-19]. Long-term ADV and TDF treatment were both associated with proximal RTD in $15 \%$ of patients [19]. The inadequacies of these studies were small sample size, insufficient assessment of proximal renal tubular function, or retrospective conducted studies [15-19]. Due to the limitation of previous reports in term of lowquality of data and the short duration of follow-up, the recent guideline of the American Association for the Study of Liver Diseases (AASLD) recommends no preference between ETV and TDF regarding possible long-term risks of renal and bone complications [7]. The available literatures are casting doubt on the long-term safety of nucleotide analogue treatment in $\mathrm{CHB}$ patients.

In order to evaluate the possible risk of nucleotide analogues, we systematically assessed the prevalence, clinical presentation, and risk factors of nucleotide analogue-related RTD in CHB patients, who were treated with long-term nucleotide analogues.

\section{Materials and Methods}

2.1. Patients. A cross-sectional study was conducted at the liver clinics of Ramathibodi Hospital, a tertiary care center, between 1 June 2014 and 31 March 2015. The study was approved by the Committee on Human Rights related to Research Involving Human Subjects, Faculty of Medicine, Ramathibodi Hospital, Mahidol University. The study was performed according to the 1975 Declaration of Helsinki. Informed consent was obtained from the subjects before enrollment. $\mathrm{CHB}$ patients with age over 18 years, having received nucleotide analogues (e.g., ADV and TDF) as monoor add-on therapy for more than 1 year and having serum creatinine level less than $1.5 \mathrm{mg} / \mathrm{dL}$, were enrolled consecutively. Exclusion criteria included pregnancy, decompensated cirrhosis, coinfected with human immune deficiency virus or hepatitis $\mathrm{C}$ virus, and the presence of glomerular or tubulointerstitial diseases secondary to poorly controlled conditions such as type 2 diabetes, hypertension. Clinical information was obtained from medical records and the hospital database.

2.2. Sample Collection and Laboratory Testing. Fasting serum, spot, and 24-hour urine samples were collected after informed consent was obtained. Fasting serum samples were assayed for liver function test, glucose, blood urea nitrogen (BUN), creatinine, electrolyte, phosphate, and uric acid. Twenty-four-hour urine was tested for protein, creatinine, potassium, bicarbonate, phosphate, and uric acid. Urinalysis with dipstick and microscopy was performed in random urine samples. Urine protein to creatinine ratio (UPCR) was obtained from the ratio of random urine protein and creatinine [20, 21]. CKD-EPI equation was used to represent an estimated glomerular filtration rate (GFR), which was derived from GFR $=141 \times \min (\text { creatinine } / \kappa, 1)^{\alpha} \times$ $\max (\text { creatinine } / \kappa, 1)^{-1.209} \times 0.993^{\text {Age }} \times 1.018$ [if female]. In this equation, $\kappa$ is 0.7 for females and 0.9 for males, $\alpha$ is -0.329 for females and -0.411 for males, min indicates the minimum of creatinine $/ \kappa$ or 1 , and max indicates the maximum of serum creatinine $/ \kappa$ or 1 [22]. Fractional excretion of potassium $($ FEK) $([$ urine potassium $\times$ plasma creatinine]/[urine creatinine $\times$ plasma potassium $] \times 100$ ), fractional excretion of phosphate $\left(\mathrm{FEPO}_{4}\right)$ ([urine phosphate $\times$ plasma creatinine]/[urine creatinine $\times$ plasma phosphate] $\times 100$ ), and fractional excretion of uric acid (FEUA) ([urine uric acid $\times$ plasma creatinine]/[urine creatinine $\times$ plasma uric acid] $\times$ 100) were calculated from serum and 24 -hour urine samples $[20,21,23]$. Tubular maximal reabsorption rate of phosphate to GFR ( $\mathrm{TmPO}_{4} / \mathrm{GFR}$ ) (plasma $\mathrm{PO}_{4}$ - ([urine phosphate $\times$ plasma creatinine]/urine creatinine)) was calculated [18].

\subsection{Definition and Criteria of Proximal RTD [17, 23, 24]}

(i) Proteinuria $=24$-hour urine protein $>150 \mathrm{mg}$

(ii) Glycosuria with normoglycemia = positive glucose dipstick (or urine glucose $>300 \mathrm{mg}$ per day) while fasting glucose $<100 \mathrm{mg} / \mathrm{dL}$

(iii) Phosphaturia $=\mathrm{FEPO}_{4}>18 \%$ or 24 -hour urine phosphate $>1,200 \mathrm{mg}$

(iv) Uricosuria $=$ FEUA $>15 \%$

(v) Renal potassium loss = hypokalemia with FEK $>6.5 \%$ or 24-hour urine potassium $>20 \mathrm{mEq}$ per day

(vi) Renal tubular acidosis = serum bicarbonate $<19 \mathrm{mmol} / \mathrm{L}$ with normal gap acidosis.

Subclinical proximal RTD was defined when 2 criteria presented, and overt proximal RTD was defined when $\geq 3$ criteria were identified. 
TABLE 1: Demographics of 92 patients on nucleotide analogues according to the severity of proximal renal tubular dysfunction (RTD).

\begin{tabular}{|c|c|c|c|c|}
\hline & Normal $(n=68)$ & Subclinical proximal RTD $(n=15)$ & Overt proximal RTD $(n=9)$ & $P$ \\
\hline Age (years) & $54.0 \pm 8.8$ & $56.2 \pm 12.5$ & $60.2 \pm 15.5$ & 0.21 \\
\hline Male, $n(\%)$ & $43(63.2)$ & $11(73.3)$ & $5(55.6)$ & 0.65 \\
\hline Liver cirrhosis, $n(\%)$ & $21(30.9)$ & $4(26.7)$ & $3(33.3)$ & 0.93 \\
\hline Type 2 diabetes, $n(\%)$ & $12(17.6)$ & $3(20.0)$ & $2(22.2 \%)$ & 0.93 \\
\hline Hypertension, $n(\%)$ & $20(29.4)$ & $8(53.3)$ & $3(33.3)$ & 0.21 \\
\hline Duration of nucleotide analogue taking (months) & $50.7 \pm 28.9$ & $65.1 \pm 30.2$ & $78.7 \pm 20.2$ & 0.01 \\
\hline
\end{tabular}

Data are expressed as mean \pm SD unless otherwise indicated.

2.4. Statistical Analysis. From a previous study, we assumed a prevalence of nucleotide analogue related proximal RTD to be $15 \%$ [19]. Based on this prevalence, the required sample size was computed using OpenEpi online software to be 83 assuming a confidence limit of $7.5 \%$ and $\alpha$ level of 0.05 [25]. Eighty-three patients were required for enrollment. Data are expressed as mean \pm standard deviation (SD) or median (range). Categorical variables were compared with Chi-square or Fisher's exact tests. Means and medians were analyzed by one-way analysis of variance (ANOVA) and nonparametric test, respectively. Statistical analysis was performed using SPSS version 16.0 (Chicago, IL).

\section{Results}

3.1. Data of CHB Patients on Nucleotide Analogue Treatment Based on the Severity of Proximal RTD (Table 1). During the study period, $110 \mathrm{CHB}$ patients who were on nucleotide analogues were screened. Fifteen patients were excluded due to the presence of significant comorbidities, for example, diabetes mellitus and hypertension. Three patients refused to participate with the study due to their personal reasons. Ninety-two CHB patients were enrolled. The mean age was $55.0 \pm 10.3$ years. Fifty-nine $(64.1 \%)$ patients were male, with compensated cirrhosis in $28(30.4 \%)$ patients. The mean duration of nucleotide analogue usage was $55.8 \pm 29.6$ (range 12-116) months. Type 2 diabetes and hypertension were present in $17(18.5 \%)$ and 31 (33.7\%) patients. The mean serum creatinine level was $0.97 \pm 0.2$ (range $0.49-1.50) \mathrm{mg} / \mathrm{dL}$. The details of current antiviral drugs were as follows: ADV in $3(3.3 \%)$ patients, TDF in $29(31.5 \%)$ patients, ADV add-on LAM (ADV-LAM) in $11(12.0 \%)$ patients, and TDF add-on LAM (TDF-LAM) in 49 (53.2\%) patients. Among 78 patients who were on TDF regimen (TDF and TDF-LAM), 27 had been switched from ADV because of financial problems or inadequate virological response.

Subclinical and overt proximal RTD were detected in 15 $(16.3 \%)$ and $9(9.8 \%)$ patients. The mean age, the number of type 2 diabetes, and hypertension among the normal, subclinical, and overt proximal RTD groups were not different. The patients with subclinical and overt proximal RTD had longer duration of nucleotide analogue treatment $(P=0.01)$. Eleven out of $78(14.1 \%)$ patients with TDF regimen and 4 out of 14 (28.6\%) patients with ADV regimen (ADV and ADV-LAM) developed subclinical proximal RTD. Six (7.7\%) patients with TDF-LAM and 3 (21.4\%) patients with ADV regimen had overt proximal RTD. Thus, proximal RTD was found in 17
(21.9\%) patients in the TDF regimen and 7 (50\%) patients in the ADV regimen. Moreover, proximal RTD occurred in 7 out of 51 patients with TDF who never received ADV and 7 out of 13 patients with ADV who were never exposed to TDF.

Among 24 patients who were found to have proximal RTD, all but one patient who was classified as having overt proximal RTD were asymptomatic. Muscle weakness and bone pain were two complaints of this patient while severe hypophosphatemia was detected. His bone mineral density revealed osteoporosis. Bone mineral density was done in 15 patients with proximal RTD. Osteoporosis (6 patients) and osteopenia (4 patients) were found in $10(66.7 \%)$ patients.

While the severity of proximal RTD worsened, urinary protein increased and renal function deteriorated progressively (Table 2). Tubular maximal reabsorption rate of phosphate to GFR ( $\left.\mathrm{TmPO}_{4} / \mathrm{GFR}\right)$ was gradually decreasing $(P<$ $0.001)$. Serum phosphate and uric acid levels diminished in higher degree of proximal RTD $(P<0.001$ for both) (Figure 1(a)). The lower serum phosphate and uric acid levels were explained by progressively increased renal phosphate and uric acid losses in subclinical and overt proximal RTD $(P<$ 0.001 for both) as shown in Figure 1(b). Increase in urinary potassium was observed in higher degree of proximal RTD $(P=0.03)$ despite unchanged serum potassium level $(P=$ $0.16)$. None of the patients in this study had hypokalemia from renal potassium losses. Glycosuria with normoglycemia was seen in $4(44.4 \%)$ patients in the overt proximal RTD group. Normal gap acidosis occurred in one case in the overt proximal RTD group. Although liver enzymes were overall normal, serum alkaline phosphatase level was significantly elevated in the proximal RTD groups (Table 2).

3.2. The Advantage of Using the Proximal RTD Criteria to Diagnose Renal Tubular Dysfunction in Patients with Subclinical Proximal RTD in Comparison with the Recommendation of the AASLD Guideline [7, 17, 23, 24]. For the followup of proximal tubular function during the treatment with nucleotide analogues, the AASLD guideline suggests that serum phosphate, urine glucose, and urine protein should be performed periodically [7]. We took the opportunity to look into this issue. Out of 83 patients who did not have overt proximal RTD, 15 (18.1\%) patients were found to have subclinical RTD by the proximal RTD criteria, comparing to $5(6.0 \%), 4(4.8 \%)$, and $4(4.8 \%)$ patients who were identified to have hypophosphatemia, glycosuria by positive glucose 
TABLE 2: Blood and urine chemistries of 92 patients treated with nucleotide analogues according to the severity of proximal renal tubular dysfunction (RTD).

\begin{tabular}{|c|c|c|c|c|}
\hline & Normal $(n=68)$ & Subclinical proximal RTD $(n=15)$ & Overt proximal RTD $(n=9)$ & $P$ \\
\hline $\operatorname{AST}(\mathrm{IU} / \mathrm{L})$ & $38.9 \pm 21.7$ & $34.4 \pm 9.3$ & $29.1 \pm 7.6$ & 0.30 \\
\hline $\operatorname{ALT}(\mathrm{IU} / \mathrm{L})$ & $53.8 \pm 35.8$ & $50.7 \pm 26.9$ & $35.7 \pm 7.7$ & 0.31 \\
\hline $\operatorname{ALP}(\mathrm{IU} / \mathrm{L})$ & $74.7 \pm 18.8$ & $89.3 \pm 28.4$ & $107.9 \pm 60.3$ & 0.036 \\
\hline Creatinine $(\mathrm{mg} / \mathrm{dL})$ & $0.92 \pm 0.2$ & $1.05 \pm 0.2$ & $1.19 \pm 0.2$ & 0.002 \\
\hline GFR by CKD-EPI (mL/min) & $86.7 \pm 16.6$ & $74.5 \pm 16.0$ & $59.9 \pm 13.6$ & $<0.001$ \\
\hline Proteinuria, $n(\%)$ & $9(13.2)$ & $10(66.7)$ & $9(100)$ & $<0.001$ \\
\hline Phosphaturia, $n(\%)$ & $9(13.2)$ & $13(86.7)$ & $9(100)$ & $<0.001$ \\
\hline Uricosuria, $n(\%)$ & 0 & $5(33.3)$ & $9(100)$ & $<0.001$ \\
\hline Glycosuria with normoglycemia, $n(\%)$ & $2(2.9)$ & $2(13.3)$ & $4(44.4)$ & $<0.001$ \\
\hline Renal potassium loss, $n(\%)$ & 0 & 0 & 0 & \\
\hline Normal gap acidosis, $n(\%)$ & 0 & 0 & $1(11.1)$ & 0.009 \\
\hline Median 24-hour urine protein (mg) & $86(0-425)$ & $158(55-437)$ & $408(190-939)$ & $<0.001$ \\
\hline Median UPCR (mg/mg) & $0.10(0-0.49)$ & $0.18(0.08-0.42)$ & $0.54(0.29-1.14)$ & $<0.001$ \\
\hline $\mathrm{TmPO}_{4} / \mathrm{GFR}(\mathrm{mg} / \mathrm{dL})$ & $2.9 \pm 0.5$ & $2.3 \pm 0.3$ & $1.7 \pm 0.7$ & $<0.001$ \\
\hline
\end{tabular}

AST, aspartate aminotransferase; ALT, alanine aminotransferase; ALP, alkaline phosphatase; GFR, glomerular filtration rate; UPCR, urine protein to creatinine ratio; $\mathrm{TmPO}_{4} / \mathrm{GFR}$, tubular maximal reabsorption rate of phosphate to GFR.

Data are expressed as mean \pm SD unless otherwise indicated.

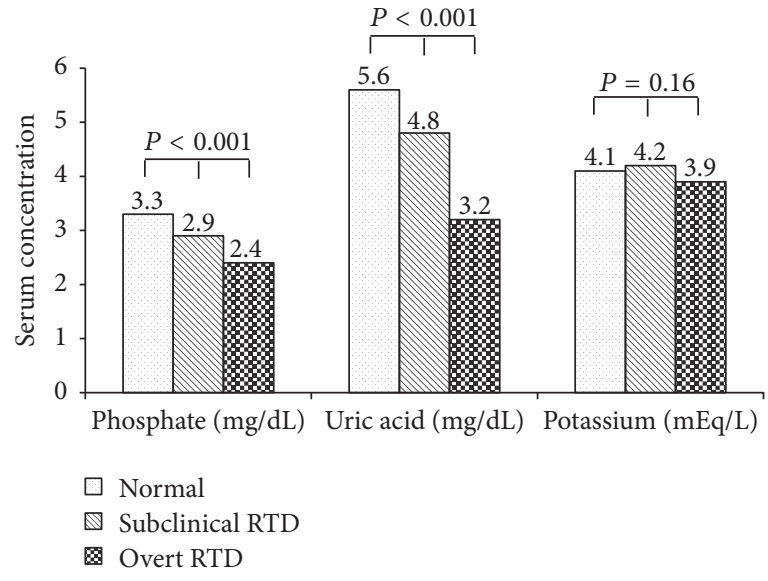

(a)
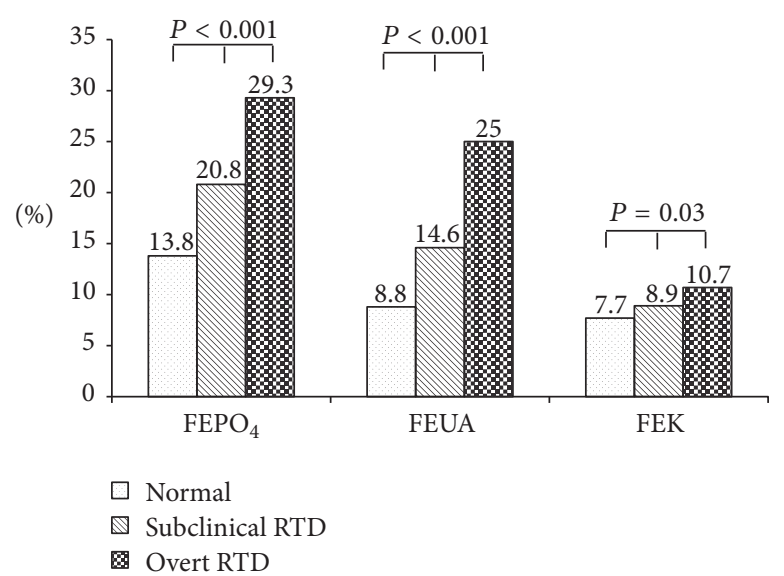

$\mathbb{Q}$ Subclinical RTD

Overt RTD

FIGURE 1: Serum phosphate, uric acid, and potassium levels among 3 groups are showed in (a). Fractional excretion of phosphate, uric acid, and potassium among 3 groups are showed in (b). FEPO, fractional excretion of phosphate; FEUA, fractional excretion of uric acid, and FEK, fractional excretion of potassium.

dipstick $(\geq 1+)$, and proteinuria by positive protein dipstick ( $\geq$ trace), respectively (Figure 2 ).

\subsection{Improvement of Proximal Renal Tubular Function at} 3 Months after Discontinuation of Nucleotide Analogues (Table 3). Nucleotide analogues were discontinued after proximal RTD was detected. In total 9 patients in the overt proximal RTD and 8 patients in the subclinical proximal RTD groups were enrolled in our on-going and follow-up study of renal tubular function after discontinuation of nucleotide analogues, and antiviral regimens were changed to ETV or LAM monotherapy. Osteoporosis was treated with bisphosphonate. Calcium carbonate and vitamin $\mathrm{D}$ were given to the patients with osteoporosis or osteopenia. Three months after nucleotide discontinuation, a full recovery of proximal RTD was seen in $5(29.4 \%)$ patients. Mean GFR increased from $67.8 \pm 17.0$ to $74.6 \pm 16.3 \mathrm{~mL} / \mathrm{min}(P=0.005)$ with a reduction of median 24-hour urine protein from 252 (55-939) to 88.5 (0-355) $\mathrm{mg}(P=0.003)$. Serum phosphate and uric acid levels increased significantly at 3 months after drug discontinuation while renal loss of uric acid was significantly decreased, with a marginal decline in renal phosphate loss.

\section{Discussion}

After long-term nucleotide analogue treatment in CHB, 26\% of patients developed proximal RTD in this study. Overt proximal RTD was seen in an estimated $10 \%$ of patients. The 
TABLE 3: Blood and urine chemistries at baseline and 3 months after discontinuation of nucleotide analogues in 17 patients with proximal renal tubular dysfunction (RTD).

\begin{tabular}{lccc}
\hline & At baseline & Three months after drug discontinuation & $P$ \\
\hline Creatinine $(\mathrm{mg} / \mathrm{dL})$ & $1.04 \pm 0.2$ & $0.96 \pm 0.2$ & 0.005 \\
GFR by CKD-EPI (mL/min) & $67.8 \pm 17.0$ & $74.6 \pm 16.3$ & 0.005 \\
Serum phosphate (mg/dL) & $2.5 \pm 0.6$ & $3.1 \pm 0.6$ & 0.002 \\
Serum uric acid (mg/dL) & $3.4 \pm 1.1$ & $3.9 \pm 1.0$ & $<0.001$ \\
Serum potassium (mEq/L) & $4.0 \pm 0.3$ & $4.0 \pm 0.3$ & 0.53 \\
FEPO $_{4}(\%)$ & $25.5 \pm 9.0$ & $22.5 \pm 12.1$ & 0.13 \\
FEUA (\%) & $22.9 \pm 9.2$ & $18.1 \pm 8.3$ & 0.003 \\
FEK (\%) & $9.8 \pm 4.7$ & $13.7 \pm 14.7$ & 0.40 \\
Median 24-hour urine protein $(\mathrm{mg})$ & $252(55-939)$ & $88.5(0-355)$ & 0.003 \\
\hline
\end{tabular}

GFR, glomerular filtration rate; $\mathrm{FEPO}_{4}$, fractional excretion of phosphate; FEUA, fractional excretion of uric acid; FEK, fractional excretion of potassium.

Data are expressed as mean \pm SD unless otherwise indicated.

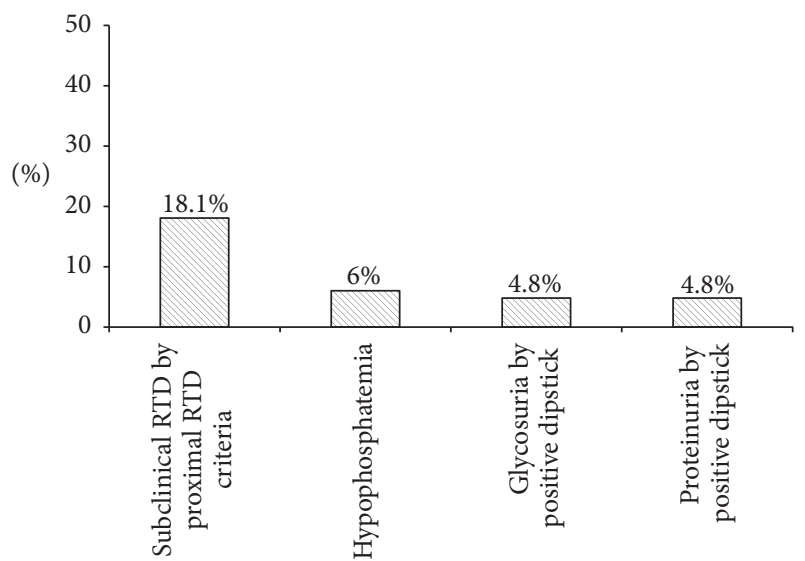

FIGURE 2: The percentage of subclinical renal tubular dysfunction defined by the proximal renal tubular dysfunction (RTD) criteria, hypophosphatemia, glycosuria, and proteinuria from positive urinary dipstick.

prevalence of nucleotide analogue-related proximal RTD in our study was higher than a previous report (15\%), which may be due to the different criteria of proximal RTD in our study $[17,23,24]$ and the other report [19]. On contrary, previous studies did not report significant reduction in renal function after long-term nucleotide analogue treatment in CHB $[13-15,26]$. However, comprehensive laboratory testing for proximal renal tubular function was not performed in these reports. Forty-one (44.6\%) patients currently received ADV (14 patients) or used to receive ADV prior to the switch to TDF (27 patients). Higher rates of renal impairment and Fanconi syndrome have been more reported with ADV treatment than TDF [12]. Long-term studies of CHB patients receiving TDF revealed no clinically relevant changes in renal function [13-15]. The greater number of proximal RTD in this study may be explained from the high number of ADV exposure. The issue of multiple and heterogeneous regimens of nucleotide analogue treatment in mono- and add-on therapy with LAM is one of the limitations of this study. The duration of nucleotide analogue treatment in our study (55.8 \pm 29.6 months) was longer than those in previous studies
(24 months and 2.4 years) $[15,26]$. These results indicated that long-term nucleotide analogue treatment could cause renal damage unless the drugs had been timely withdrawn. The longstanding use of ADV and TDF can increase some risk of proximal RTD. It is worthy of note that TDF-related proximal RTD can occur in the absence of rising serum creatinine level $[23,27]$. All, but one patient in this study with overt proximal RTD, presented without symptom. Thus, the presence of nucleotide analogue-related proximal RTD might not be clinically recognized unless the careful evaluation of renal tubular function was carried out. Furthermore, twentyhour urine collection was used for the estimation of protein, phosphate, and uric acid losses instead of spot urine samples for more accurate results. From our final analysis, there was insignificant difference in the results of spot and 24hour urine samples. Spot urine samples can be used for the monitoring of renal tubular function in clinical practice. From the AASLD guideline, serum creatinine, phosphate, urine glucose, and urine protein should be evaluated before initiation of TDF and periodically thereafter [7]. We have showed that the proximal RTD criteria performed better than the testing of serum phosphate and urine analysis with urinary dipstick to identify patients with subclinical tubular dysfunction.

In this study, phosphaturia was the most common feature when proximal RTD was described. $\mathrm{FEPO}_{4}>18 \%$ was used for the criteria of tubular phosphate loss in our study [24], while tubular reabsorption of phosphate (TRP) $<82 \%$ was an alternative criterion used in some other reports $[19,23$, 28]. In addition, the problem of severe tubular phosphate loss in subclinical and overt proximal RTD was confirmed with low $\mathrm{TmPO}_{4} / \mathrm{GFR}(<2.8 \mathrm{mg} / \mathrm{dL})$ in our study. Nucleotide analogue-related Fanconi syndrome, which is an extreme degree of proximal RTD, has a particularly low incidence in $\mathrm{CHB}$ treatment as shown from a few case reports [29, 30]. In this study, acid loading test and the investigation of aminoaciduria were not done to confirm the diagnosis of Fanconi syndrome, although some patients with overt proximal RTD shared many features of Fanconi syndrome. Osteoporosis and osteopenia were found in 10 (66.7\%) patients who had proximal RTD, which was very close to the prevalence of the reduction of bone mineral density 
in patients who were treated with nucleotide analogues in a previous report [31]. Long-term hypophosphatemia with decreased renal phosphate absorption can lead to osteomalacia and impaired bone health [29].

In long-term $\mathrm{ADV}$ usage in $\mathrm{CHB}$ treatment, age and the duration of ADV usage were the predictors of the development of proximal RTD [17]. The other study revealed that preexisting renal dysfunction was the predisposing factor of nucleotide analogue-related proximal RTD in patients with CHB [19]. From our study, the duration of nucleotide analogue was the only significant factor associated with proximal RTD. Age was not found to be a risk factor, which may be due to the lower number of older patients in this study. TDF is eliminated unchanged in urine by glomerular filtration and proximal tubular secretion. About $20-30 \%$ of TDF is absorbed at proximal tubule cells through basal membrane organic anion transporter (hOAT1 and hOAT3) and exits across apical membrane via multidrug resistant-associated protein 4 (MRP4) and MRP2 [27]. Polymorphism of genes (ABCC4 and ABCC2) encoding these transporters can result in TDF-related proximal RTD [27]. Hence, proximal RTD can occur in an individual, but not in others who are treated with the same nucleotide analogue [27].

Once proximal RTD occurs, TDF should be discontinued and replaced with other antiviral drugs with consideration for the previous history of $\mathrm{HBV}$ drug resistance [7]. The proximal renal tubular function needs to be followed up closely. If there is recovery of proximal RTD after discontinuation of nucleotide analogues, it will help to confirm that the proximal RTD is related to long-term nucleotide analogue treatment [10]. A full recovery of renal tubular function was seen in about $29 \%$ at three months after discontinuation of nucleotide analogues in this study. The result of long-term outcome of discontinuation of nucleotide analogues still remains to be investigated. Therefore, early detection of nucleotide analogue-related proximal RTD and early withdrawal of the drug are the fundamental approaches to avoid irreversible proximal renal tubular injury [10]. The role of new nucleotide analogues without nephrotoxicity such as tenofovir alafenamide (TAF) in the prevention and reduction of nucleotide analogue-related nephrotoxicity is under investigation [32].

There are some limitations of our study that need to be addressed. It was a single center and cross-sectional study which lacked the baseline data of patients before the initiation of nucleotide analogues. As a tertiary center, patients with more severe liver diseases and multiple medical conditions were enrolled to the study. During the course of treatment, nucleotide analogue was switched in some patients due to problems such as financial issues and inadequate virological response.

\section{Conclusion}

Our study has demonstrated that proximal RTD and a decline in GFR are associated with long-term nucleotide analogue treatment in $\mathrm{CHB}$ patients. Comprehensive laboratory testing is the crucial step for the early detection of proximal RTD to avoid irreversible renal dysfunction. Genetic studies in the polymorphisms of drug transporters should be investigated to determine their roles in this condition. Further welldesigned multicenter studies should be carried out on a larger scale basis.

\section{Abbreviations}

CHB: Chronic hepatitis B

RTD: Renal tubular dysfunction

LAM: Lamivudine

$\mathrm{FEPO}_{4}$ : Fractional excretion of phosphate

FEUA: Fractional excretion of uric acid

FEK: Fractional excretion of potassium

HBV: Hepatitis B virus

TDF: Tenofovir disoproxil fumarate

ADV: Adefovir dipivoxil

UPCR: Urine protein to creatinine ratio

GFR: Glomerular filtration rate

TAF: Tenofovir alafenamide.

\section{Competing Interests}

The authors declare that there is no conflict of interests regarding the publication of this paper.

\section{Authors' Contributions}

All authors contributed substantially to this work and gave approval to the final draft.

\section{Acknowledgments}

The study was supported by Faculty of Medicine, Ramathibodi Hospital, Mahidol University, and the Gastroenterological Association of Thailand.

\section{References}

[1] G. Fattovich, F. Bortolotti, and F. Donato, "Natural history of chronic hepatitis B: special emphasis on disease progression and prognostic factors," Journal of Hepatology, vol. 48, no. 2, pp. 335$352,2008$.

[2] U. H. Iloeje, H.-I. Yang, J. Su, C.-L. Jen, S.-L. You, and C.-J. Chen, "Predicting cirrhosis risk based on the level of circulating hepatitis B viral load," Gastroenterology, vol. 130, no. 3, pp. 678686, 2006.

[3] C.-J. Chen, H.-I. Yang, J. Su et al., "Risk of hepatocellular carcinoma across a biological gradient of serum hepatitis B virus DNA Level," The Journal of the American Medical Association, vol. 295, no. 1, pp. 65-73, 2006.

[4] Y.-F. Liaw, J. J. Y. Sung, W. C. Chow et al., "Lamivudine for patients with chronic hepatitis B and advanced liver disease," The New England Journal of Medicine, vol. 351, no. 15, pp. 15211531, 2004.

[5] European Association for the Study of the Liver, "EASL clinical practice guidelines: management of chronic hepatitis B virus infection," Journal of Hepatology, vol. 57, no. 1, pp. 167-185, 2012.

[6] T. Asselah and P. Marcellin, "Long-term results of treatment with nucleoside and nucleotide analogues (entecavir and tenofovir) for chronic hepatitis B," Clinics in Liver Disease, vol. 17, no. 3, pp. 445-450, 2013. 
[7] N. A. Terrault, N. H. Bzowej, K.-M. Chang, J. P. Hwang, M. M. Jonas, and M. H. Murad, "AASLD guidelines for treatment of chronic hepatitis B," Hepatology, vol. 63, no. 1, pp. 261-283, 2016.

[8] M. C. Segovia, W. Chacra, and S. C. Gordon, "Adefovir dipivoxil in chronic hepatitis B: history and current uses," Expert Opinion on Pharmacotherapy, vol. 13, no. 2, pp. 245-254, 2012.

[9] J. Fung, C.-L. Lai, W.-K. Seto, and M.-F. Yuen, "Nucleoside/ nucleotide analogues in the treatment of chronic hepatitis B," Journal of Antimicrobial Chemotherapy, vol. 66, no. 12, pp. 27152725, 2011.

[10] B. Fernandez-Fernandez, A. Montoya-Ferrer, A. B. Sanz et al., "Tenofovir nephrotoxicity: 2011 update," AIDS Research and Treatment, vol. 2011, Article ID 354908, 11 pages, 2011.

[11] H. Izzedine, J. S. Hulot, V. Launay-Vacher et al., "Renal safety of adefovir dipivoxil in patients with chronic hepatitis B: two double-blind, randomized, placebo-controlled studies," Kidney International, vol. 66, no. 3, pp. 1153-1158, 2004.

[12] J. Fung, W.-K. Seto, C.-L. Lai, and M.-F. Yuen, "Extrahepatic effects of nucleoside and nucleotide analogues in chronic hepatitis B treatment," Journal of Gastroenterology and Hepatology, vol. 29, no. 3, pp. 428-434, 2014.

[13] M. Buti, N. Tsai, J. Petersen et al., "Seven-year efficacy and safety of treatment with tenofovir disoproxil fumarate for chronic hepatitis B virus infection," Digestive Diseases and Sciences, vol. 60, no. 5, pp. 1457-1464, 2015.

[14] M. Buti, S. Fung, E. Gane et al., "Long-term clinical outcomes in cirrhotic chronic hepatitis B patients treated with tenofovir disoproxil fumarate for up to 5 years," Hepatology International, vol. 9, no. 2, pp. 243-250, 2015.

[15] S. Koklu, M. T. Gulsen, Y. Tuna et al., "Differences in nephrotoxicity risk and renal effects among anti-viral therapies against hepatitis B," Alimentary Pharmacology and Therapeutics, vol. 41, no. 3, pp. 310-319, 2015.

[16] M. Tanaka, F. Suzuki, Y. Seko et al., "Renal dysfunction and hypophosphatemia during long-term lamivudine plus adefovir dipivoxil therapy in patients with chronic hepatitis B," Journal of Gastroenterology, vol. 49, no. 3, pp. 470-480, 2014.

[17] M. Shimizu, N. Furusyo, H. Ikezaki et al., "Predictors of kidney tubular dysfunction induced by adefovir treatment for chronic hepatitis B," World Journal of Gastroenterology, vol. 21, no. 7, pp. 2116-2123, 2015.

[18] C. Tien, J. J. Xu, L. S. Chan et al., "Long-term treatment with tenofovir in asian-american chronic hepatitis B patients is associated with abnormal renal phosphate handling," Digestive Diseases and Sciences, vol. 60, no. 2, pp. 566-572, 2015.

[19] N. Gara, X. Zhao, M. T. Collins et al., "Renal tubular dysfunction during long-term adefovir or tenofovir therapy in chronic hepatitis B," Alimentary Pharmacology and Therapeutics, vol. 35, no. 11, pp. 1317-1325, 2012.

[20] C. Chantler, "Kidney diseases in children," in Disease of the Kidney, R. Schrier and C. Gottschalk, Eds., pp. 2379-2403, Little Brown, Boston, Mass, USA, 1993.

[21] G. Fogazzi and G. Garigali, "Urinalysis," in Comprehensive Clinical Nephrology, R. Johnson, J. Feehally, and J. Floege, Eds., pp. 39-52, Elsevier Saunders, Toronto, Canada, 2015.

[22] A. S. Levey, L. A. Stevens, C. H. Schmid et al., "A new equation to estimate glomerular filtration rate," Annals of Internal Medicine, vol. 150, no. 9, pp. 604-612, 2009.

[23] P. Labarga, P. Barreiro, L. Martin-Carbonero et al., "Kidney tubular abnormalities in the absence of impaired glomerular function in HIV patients treated with tenofovir," AIDS, vol. 23, no. 6, pp. 689-696, 2009.
[24] K. Andrade-Fuentes, J. A. Mata-Marín, J. I. López-De León, B. Manjarrez-Téllez, J. L. S. Ramírez, and J. Gaytan-Martínez, "Proximal renal tubular dysfunction related to antiretroviral therapy among HIV-infected patients in an HIV clinic in Mexico," AIDS Patient Care and STDs, vol. 29, no. 4, pp. 181-185, 2015.

[25] K. M. Sullivan, A. Dean, and M. M. Soe, "OpenEpi: a web-based epidemiologic and statistical calculator for public health," Public Health Reports, vol. 124, no. 3, pp. 471-474, 2009.

[26] V. Mallet, M. Schwarzinger, A. Vallet-Pichard et al., "Effect of nucleoside and nucleotide analogues on renal function in patients with chronic hepatitis B virus monoinfection," Clinical Gastroenterology and Hepatology, vol. 13, no. 6, pp. 1181-1188, 2015.

[27] A. M. Hall, "Update on tenofovir toxicity in the kidney," Pediatric Nephrology, vol. 28, no. 7, pp. 1011-1023, 2013.

[28] E. Kinai and H. Hanabusa, "Progressive renal tubular dysfunction associated with long-term use of tenofovir DF," AIDS Research and Human Retroviruses, vol. 25, no. 4, pp. 387-394, 2009.

[29] H. J. Jeong, J. M. Lee, T. H. Lee et al., "Two cases of hypophosphatemic osteomalacia after long-term low dose adefovir therapy in chronic hepatitis B and literature review," Journal of Bone Metabolism, vol. 21, no. 1, pp. 76-83, 2014.

[30] H. S. Hwang, C. W. Park, and M. J. Song, "Tenofovir-associated Fanconi syndrome and nephrotic syndrome in a patient with chronic hepatitis B monoinfection," Hepatology, vol. 62, no. 4, pp. 1318-1320, 2015.

[31] P. Maggi, V. Montinaro, A. Leone et al., "Bone and kidney toxicity induced by nucleotide analogues in patients affected by HBV-related chronic hepatitis: a longitudinal study," Journal of Antimicrobial Chemotherapy, vol. 70, no. 4, pp. 1150-1154, 2014.

[32] X.-Y. Wang and H.-S. Chen, "Emerging antivirals for the treatment of hepatitis B," World Journal of Gastroenterology, vol. 20, no. 24, pp. 7707-7717, 2014. 


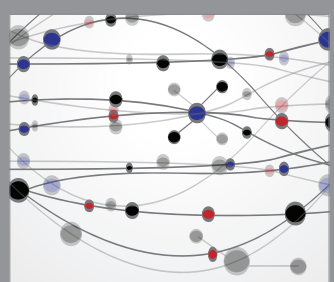

The Scientific World Journal
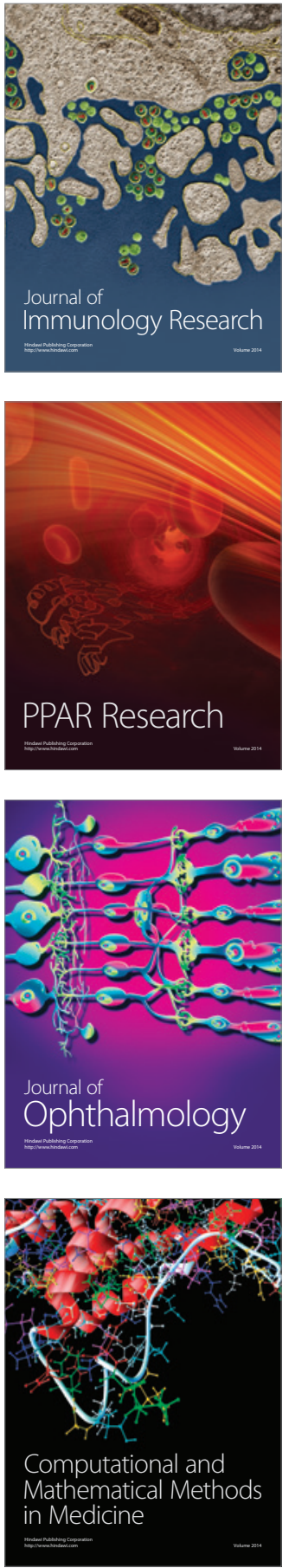

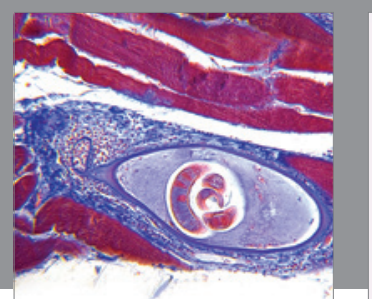

Gastroenterology Research and Practice

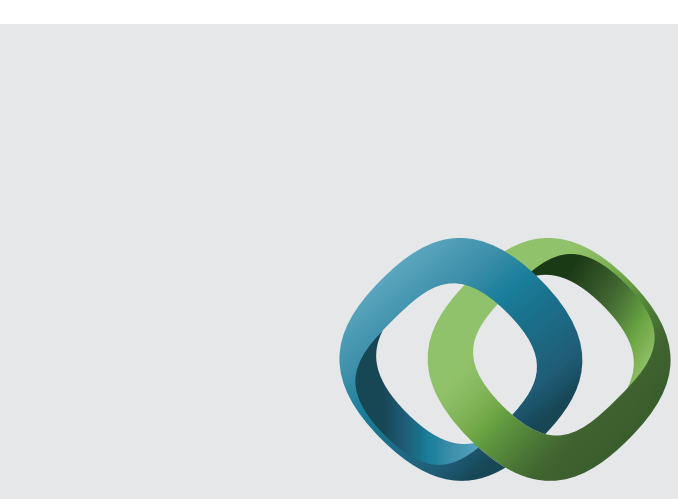

\section{Hindawi}

Submit your manuscripts at

http://www.hindawi.com
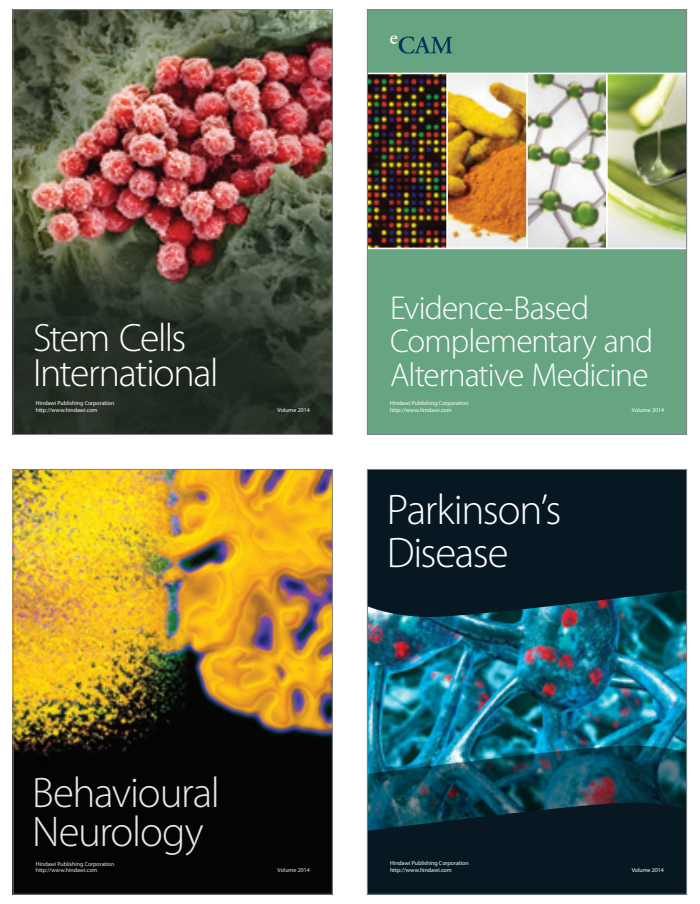
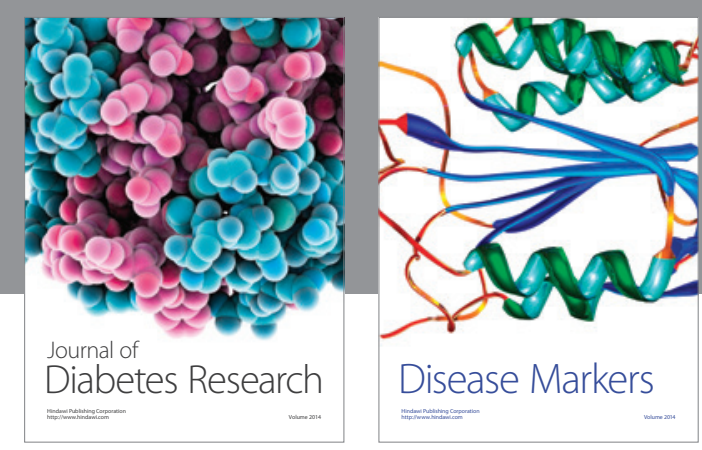

Disease Markers
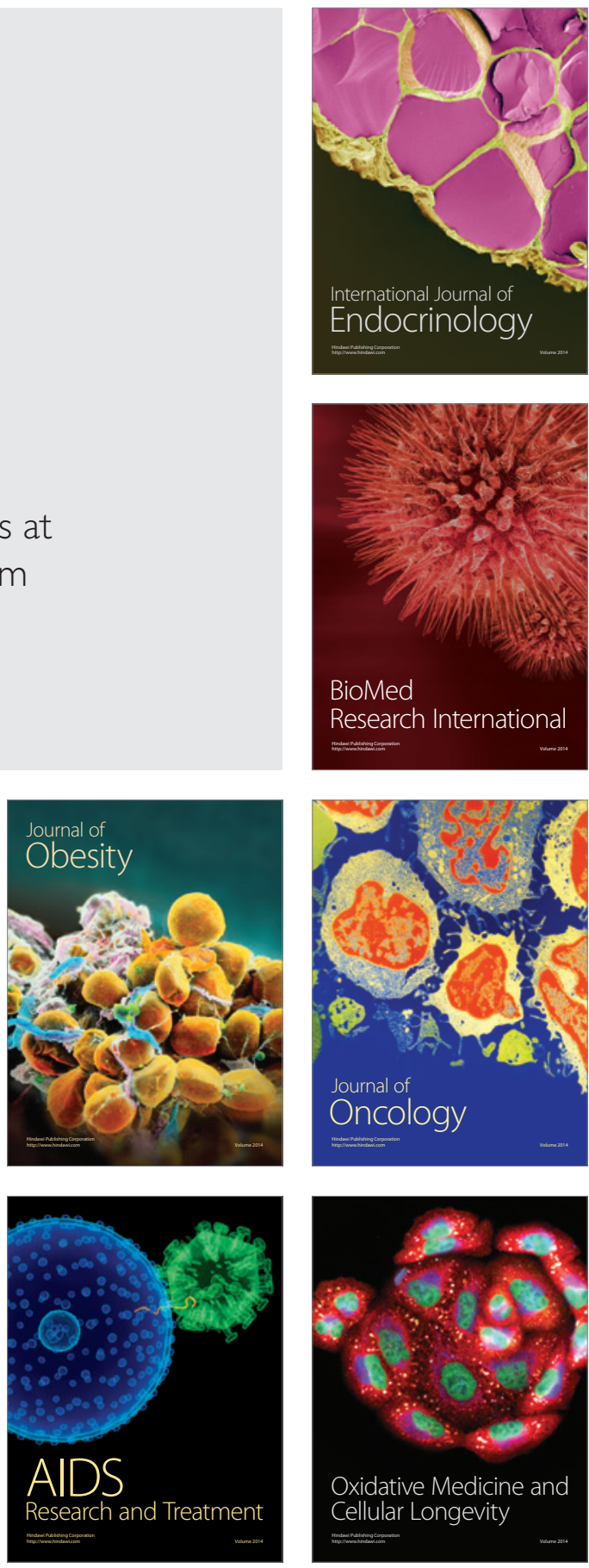